\title{
Stator elements optimization of centrifugal compressor intermediate type stage by CFD methods
}

\author{
Lyubov Marenina ${ }^{1, *}$, Yuri Galerkin ${ }^{1}$, and Alexandr Drozdov ${ }^{1}$ \\ ${ }^{1}$ Peter the Great St.Petersburg Polytechnic University, St.Petersburg, Polytechnicheskaya, 29, Russia
}

\begin{abstract}
Optimal gas-dynamic design is a complex and time-consuming process. Modern CFD methods help in solving optimization problems and reliably calculating characteristics of stator elements of centrifugal compressor stages. To carry out such calculations, it is necessary to create a parametrized model, which facilitates automation of the process of changing the flow path geometry, rebuilding its dimensions and the computational grid. Using the Direct Optimization program of the ANSYS software package, we have optimized the flow path of the stator elements of a centrifugal compressor intermediate type stage consisting of a vaneless diffuser and a return channel. In this paper, the MOGA (Multi-Objective Genetic Algorithm) optimization method was used. The object of the study was stator elements of one of the model stages designed by the Problem Laboratory of Compressor Engineering, SPbPU. The goal was to achieve the minimum value of the loss coefficient of stator elements when changing 5 geometric parameters: the number of vanes, the inlet vane angle, the height of the vane at the inlet to the return channel vane cascade, the radius of curvature of the leading edge and the thickness of the vane profile. For the best variants based on the results of optimization, the characteristics of the loss coefficient depending on the flow rate coefficient were calculated, their characteristics were compared with the initial variant of the stator elements. The best variant in the design mode has a loss coefficient $4.4 \%$ lower than the reference model. With a flow rate coefficient of 1.63 times greater than the calculated one, the optimized variant's loss coefficient is $33 \%$ less.
\end{abstract}

\section{Introduction}

The process of selection of return channel sizes and calculation of their gas-dynamic characteristics is described in the corresponding sections of the monographs [1-4] and in separate research papers [512]. Calculations of the stator elements of the flow path of the centrifugal stages by the methods of computational gas dynamics provide information, on the basis of which it is possible to formulate design recommendations. The stator elements of the model stage 0028-058-0373 (design flow coefficient 0.028, design loading factor 0.58 , hub to impeller ratio 0.373 ) by the Problem Laboratory of Compressor Engineering, SPbPU [1] were selected as the object under study. Fig. 1 shows sketches of the stage in the meridional and radial planes with the indication of the control sections and its main geometric parameters.

The goal of our work is to optimize the return channel in order to reduce the loss coefficient in the entire work area for flow rate coefficient. The set of geometric parameters that have the greatest impact on

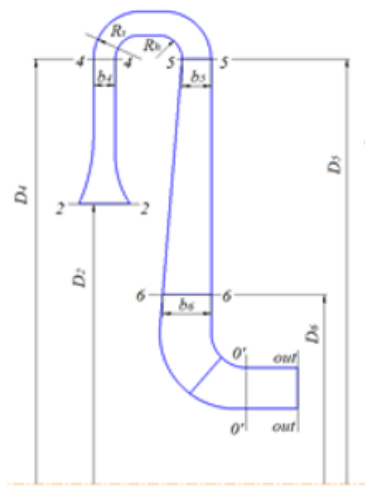

a)

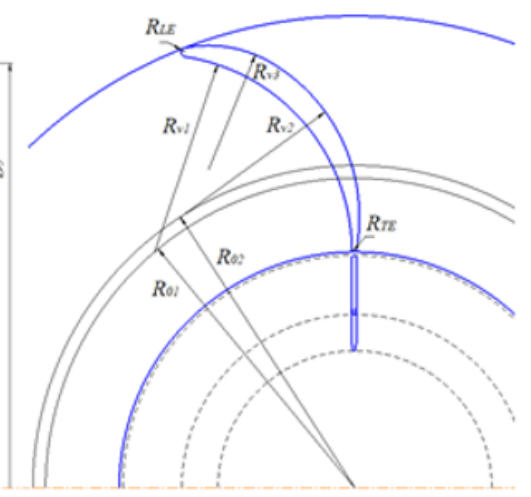

b)

Fig. 1. The stator elements sketches of the stage 0028-058-0373 in the meridional (a) and radial (b) planes.

\footnotetext{
* Corresponding author: marenina ln@ mail.ru
}

(C) The Authors, published by EDP Sciences. This is an open access article distributed under the terms of the Creative Commons Attribution License 4.0 


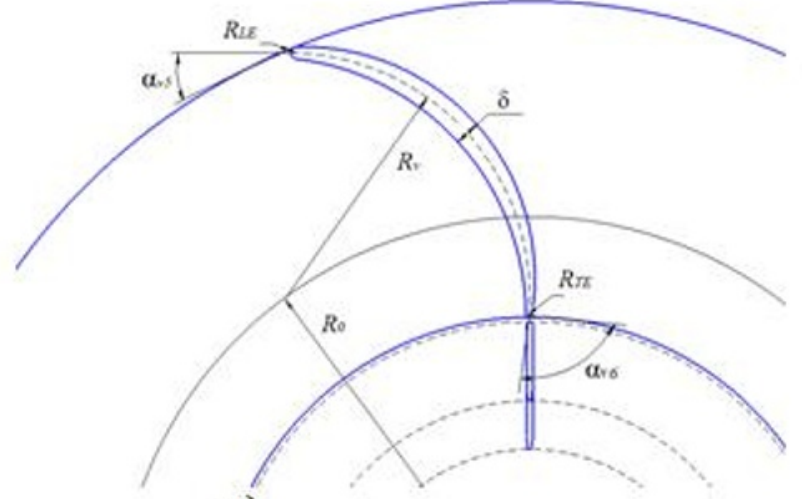

a)

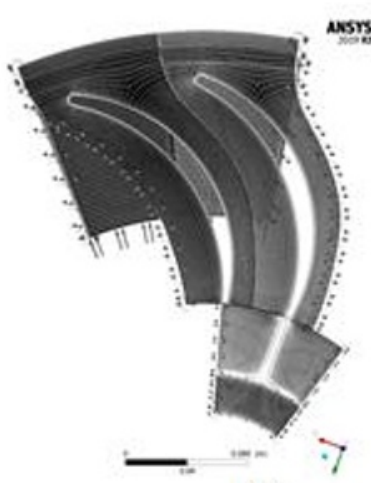

b)

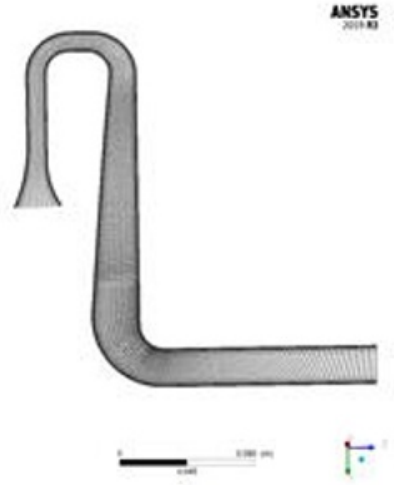

c)

Fig. 2. Vane sketch, built along the vane midline in the form of a circular arc (a), general view of the computational domain in the radial (b) and meridional (c) plane.

the shape of the flow path is optimized.

To carry out the calculations, it is necessary to create a parametrized model, which allows us to automate the processes of changing the geometry of the flow path according to its new dimensions and of rebuilding the computational grid. When constructing a sketch of the vane in the DesignModeler program, the initial vane profile was replaced with a variant made with the traditional shape of the midline of the vane in the form of a circular arc [1] (Fig. 2 a). Vanes of this shape ensured high efficiency of compressors designed by a scientific team of prof. Galerkin at SPbPU [13]. We will demonstrate the advantage of two-arc vanes compared with the profile of the model stage vanes with the calculations below. In the DesignModeler CAD program, the midline of the vanes is defined by the radii $R_{0}$ and $R_{v}$, which are calculated according to the formulas from [14]:

$$
\begin{gathered}
R_{v}=\frac{D_{6}^{2}-D_{5}^{2}}{4\left(D_{6} \cos \alpha_{v 6}-D_{5} \cos \alpha_{v 5}\right)} \\
R_{0}=\sqrt{R_{v}^{2}+0,25 D_{6}^{2}-R_{v} D_{6} \cos \alpha_{v 6}}
\end{gathered}
$$

where $D$ is a diameter, $\alpha_{v}$ is a vane angle, «5» and «6»» are indices of control sections according to the Fig. 1. The radii of the surface of the vane are automatically selected taking into account the specified radii of curvature of the leading and trailing edges $R_{L E}, R_{T E}$ and the thickness of the vane $\delta$.

\section{CFD calculation methodology}

For the correct formulation of the optimization problem, it is necessary to create a parametrized model of the flow path and determine the dimensions of the computational grid common to all calculations, select the appropriate turbulence model, and set the boundary conditions. The methodology for setting up CFD calculations was studied by the authors in $[15,16]$. At all stages of model creation and calculations, the Workbench environment of the ANSYS CFX 19 software package was used. The construction of a parameterized model of the flow path was described in detail in [17]. In DesignModeler, the calculation area is built on the basis of sketches, and all their sizes can be set in Workbench as parameters, so that when their values change, the sketch is automatically rebuilt. Also, instead of fixed parameter values, it is possible to specify expressions that describe relationships between sizes. In the TurboGrid mesh generator, design meshes for the main and straightening vanes were built. The values $\mathrm{y}^{+}<20$ were set in accordance with the requirements of the correct modeling of the boundary layer using the SST turbulence model (Shear-Stress-Transport). In the CFX-Pre program, computational grids were combined into one area consisting of two main return channel vanes and one straightening vane (Fig. $2 \mathrm{~b}, \mathrm{c}$ ). The total number of elements in the resulting area is 893000 . The following conditions were set at the boundaries:

- at the inlet: total pressure $P_{2}^{*}$, total temperature $T_{2}^{*}$ and flow direction $\alpha_{2}$;

- at the outlet: mass flow $\bar{m}$;

- on the walls from the « 0 '» section (Fig. 1) to the outlet, the «free slip» condition is accepted;

- between the grids of the main vanes, the condition «general connection»;

- between the grids of the main and straightening vanes, the condition «frozen rotor»;

- on the "periodic» surfaces, the condition of the radial periodicity «rotational periodicity» was set.

The CFD-Post program defines a formula for calculating the loss coefficient (3) as a function of the values averaged by the program in sections «2» and $\ll 0$ '».

$$
\zeta_{2-0^{\prime}}=\frac{p_{2}^{*}-p_{0^{\prime}}^{*}}{0,5\left(\rho_{2}+\rho_{0^{\prime}}\right)} \cdot \frac{2}{c_{2}^{2}}
$$

where $\rho$ is density, $c$ is velocity, and « $2 »$ and $\langle 0$ '» are the indices of control sections according to the Fig. 1. For total and static pressure, averaging over the area is used, for other values - over the mass flow rate. The 
value of this function is displayed as the output parameter of the Workbench and is used in the Direct Optimization program, where we can set the output function as the efficiency parameter, according to which the program will change the values of the input parameters to achieve the best result, in this case, the lowest value of the loss coefficient. The Direct Optimization program allows us to choose an optimization method that depends on the number of objectives, the set limits and the desired number of design points. For the research conducted in the work, the MOGA method was used. The initial conditions for the search for optimal sizes were set in accordance with the initial operating conditions of the stator elements of the stage 0028-058-0373 in the design mode with flow rate coefficient $\Phi_{d e s}=0.028$. For the initial return channel and optimized variants, the characteristic $\zeta_{2-0^{\prime}}=f(\Phi)$ was calculated at six points corresponding to the operation of the stage when it was tested with a conditional Mach number $M_{u}=0.6$. Fig. 4 shows the result of modeling the characteristics of the stage 0028058-0373 according to the IDENT program [18].

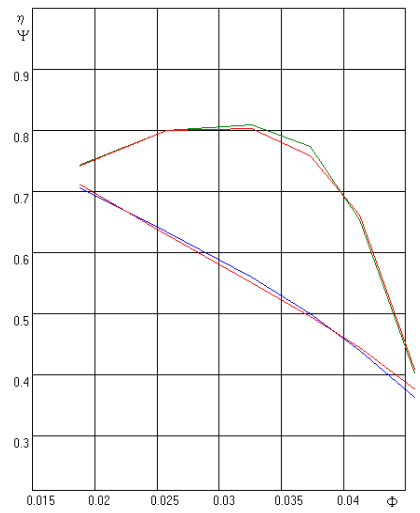

Fig. 3. The IDENT program. Stage characteristics 0028-0580373 according to the test results (green) and calculated using the Universal Modeling Method (red).

The Universal modeling method is widely used in design practice [19-23] and accurately calculates the characteristics of stages and compressors and the flow parameters in the control sections of the flow path. It is sensible to carry out our CFD study at 6 values of the flow coefficients at which the stage was tested. Table 1 shows the data from the calculation performed in the IDENT program. Since the calculation corresponding to the conditional flow rate coefficient $\Phi_{\text {des }}=0.028$ is of interest, we assume that the parameters vary linearly between their values at $\Phi=0.0326$ and 0.0258 . The data thus obtained for the design point are also listed in Table 1.

The test results of model stage 0028-058-0373 in Fig. 3 are presented in a dimensionless form. For calculations in the CFX-Pre program, the initial parameters must be specified in dimensional form. The stage was tested under the following conditions: $p_{0}^{*}=101000 \mathrm{~Pa}, T_{0}^{*}=$ $288.15 \mathrm{~K}, M_{u}=0.6$. The transition to dimensional parameters was carried out according to known ratios. The parameters are presented in Table 2 . The values of $\varphi_{2}$ are calculated according to the mathematical model when processing the results of testing the model stage that were presented in the Table 1 (above).

\section{Results and discussion}

\subsection{Optimization of the initial return channel with a two-arc profile by 5 parameters}

To carry out optimization in the design mode with $\Phi_{\text {des }}$ $=0.028$, the following geometric parameters were chosen as variable values and the limits of their change were set:

- for the number of vanes even values were set: $\mathrm{z}=$ $(18$... 28);

- for the inlet vane angle: $\alpha_{v 5}=\left(16^{\circ} \ldots 30^{\circ}\right)$;

- for the height of the vane at the inlet to the stator elements: $b_{5}=\left(\begin{array}{ll}15 & \ldots 32\end{array}\right) \mathrm{mm}$;

- for the radius of curvature of the leading edge $R_{L E}$ $=(1.4 \ldots 4) \mathrm{mm}$;

Table 1. Data from the calculation of the stage 0028-058-0373 executed in the IDENT program.

\begin{tabular}{|c|c|c|c|c|c|c|c|}
\hline No & 1 & 2 & 3 & 4 & 5 & 6 & 7 \\
\hline$\Phi$ & 0.0457 & 0.0413 & 0.0373 & 0.0326 & 0.028 & 0.0258 & 0.0187 \\
\hline$\varphi_{2}$ & 0.248 & 0.215 & 0.189 & 0.163 & 0.139 & 0.127 & 0.0925 \\
\hline$\psi_{T}$ & 0.3599 & 0.4242 & 0.4727 & 0.5238 & 0.570 & 0.5925 & 0.6601 \\
\hline$\psi_{i}$ & 0.3761 & 0.4435 & 0.4952 & 0.5508 & 0.603 & 0.6283 & 0.7114 \\
\hline
\end{tabular}

Table 2. The calculated gas-dynamic parameters necessary for setting boundary conditions.

\begin{tabular}{|c|c|c|c|c|c|c|c|}
\hline$\Phi$ & 0.0457 & 0.0413 & 0.0373 & 0.0326 & 0.028 & 0.0258 & 0.0187 \\
\hline$T_{2}^{*}, \mathrm{~K}$ & 303.75 & 306.55 & 308.69 & 311.00 & 313.18 & 314.22 & 317.66 \\
\hline$P_{2}^{*}, \mathrm{~Pa}$ & 114182 & 120813 & 125663 & 129170 & 132266 & 133981 & 136455 \\
\hline$\alpha_{2}^{0}$ & 34.6 & 26.9 & 21.8 & 17.3 & 13.8 & 12.1 & 8.0 \\
\hline $\bar{m},(\mathrm{~kg} / \mathrm{s})$ & 1.4990 & 1.3570 & 1.2260 & 1.0720 & 0.9204 & 0.8480 & 0.6147 \\
\hline
\end{tabular}



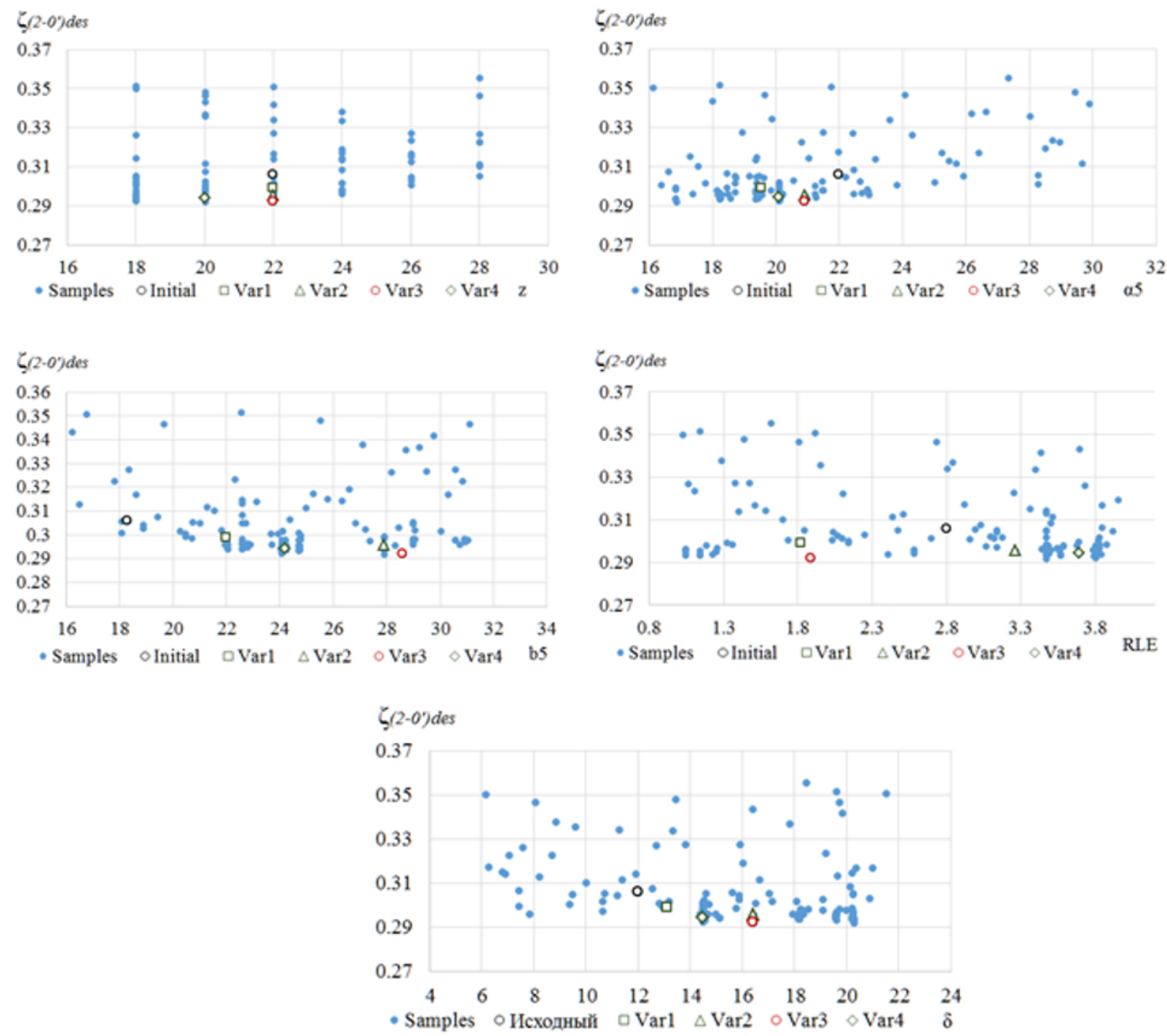

Fig. 4. ANSYS CFX 19. Results of calculation of 120 variants of stator elements obtained during optimization in the Direct Optimization program.

- for vane thickness $\delta=(6 \ldots 22) \mathrm{mm}$.

During the optimization process, 120 variants of stator elements with loss coefficient $\zeta_{2-0}$ des ranging from 0.356 to 0.292 were calculated in the Direct Optimization program. Fig. 4 presents the values of the loss coefficient for all the calculated variants for each variable parameter separately. The parameters of the four return channels with the lowest value of the loss coefficient are presented in Table 3.

The loss coefficients of optimized variants are $2.3-$ $4.5 \%$ lower than of the initial one. For the three variants, the optimal number of vanes was found to be 22 . The optimized variants have optimal vane angles ranging from 19.5 to $21^{\circ}$, which is $1-2.5^{\circ}$ less of initial, while the height of the vanes at the inlet is much greater. The biggest difference in variant 4 is that it has the maximum vane height at the inlet, almost equal to the vane height at the outlet $\left(b_{6}=30.5 \mathrm{~mm}\right)$. In addition to a smaller angle, the variant 4 profile is $36 \%$ thicker. The variant 3 has a smaller number of vanes $z=20$ and a smaller value of $b_{5}$ compared to the variant 4 . Fig. 5 presents the characteristics of the loss coefficient of the compared

Table 3. The geometric parameters of the stator elements variants considered best by optimization results in Direct Optimization.

\begin{tabular}{|l|l|l|l|l|l|l|}
\hline № & $\mathrm{z}$ & $\alpha_{v 5}$ & $b_{5}, \mathrm{~mm}\left(b_{5} / b_{4}\right)$ & $R_{L E}, \mathrm{~mm}$ & $\delta, \mathrm{mm}$ & $\zeta_{2-0^{\prime}}$ \\
\hline Initial & 22 & 22.000 & $18.300(1.34)$ & 2.800 & 12.000 & 0.3057 \\
\hline Variant 1 & 22 & 19.538 & $20.727(1.57)$ & 1.819 & 13.137 & 0.2989 \\
\hline Variant 2 & 22 & 20.862 & $27.909(2.04)$ & 3.263 & 16.454 & 0.2956 \\
\hline Variant 3 & 20 & 20.083 & $24.173(1.76)$ & 3.694 & 14.453 & 0.2943 \\
\hline Variant 4 & 22 & 20.900 & $28.600(2.09)$ & 1.819 & 16.400 & 0.2921 \\
\hline
\end{tabular}


variants.

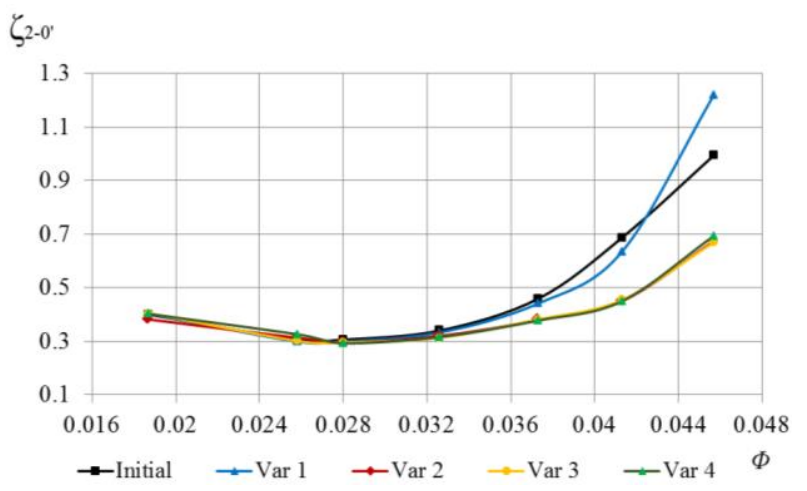

Fig. 5. ANSYS CFX 19. Loss coefficient versus flow rate coefficient.

Variant 1 does not exceed the initial variant. Variants 2, 3 and 4 are almost equivalent and noticeably more effective than the initial variant at high flow rates. With a maximum flow rate $\Phi=0.0457$, their loss coefficient is $33 \%$ less. Fig. 6 shows the flow structure in the regime with the maximum flow coefficient $\Phi=0.0457$ for the initial variant and the variant 3 . The flow in the meridional plane is more organized for the initial variant with lower diffusivity of the crossover $\left(b_{5} / b_{4}=1.34\right)$. However, in the radial plane, a more favorable flow for variant 3 is with $b_{5} / b_{4}=1.76$. At the maximum flow rate, the initial variant has a larger incidence angle, which leads to a significant separation of the flow. For the optimized variant, flow separation is virtually nonexistent.

\subsection{Verification of optimization results by CFD calculation of whole stage}

Despite considerable efforts, CFD calculations of the characteristics of centrifugal stages still do not provide reliable results $[24,25]$. The results of modeling stage 0028-058-0373 characteristics were not entirely satisfactory either [26]. The authors nevertheless believe that a comparison of the calculated characteristics of the stage with the initial and optimized return channel can confirm or refute the result of optimization. According to the authors of [26], the problem of the mismatch between the characteristics of the efficiency and the effective pressure is to a large extent connected with the fact that CFD calculations significantly overestimate the head coefficient. In order to approximate the conditions of gas movement in return channel during CFD calculation, the blade height at the outlet of the impeller was reduced. This reduced the head coefficient. Fig. 7 (left) shows the head coefficient of the impeller of the stage 0028-058-0373 in comparison with the calculation of the head coefficient of this impeller and its modification with a reduced blade height at the outlet.

For a modified impeller near the design mode $\Phi_{\text {des }}=$ 0.028, the head coefficient coincides with its actual value. In Fig. 7 (right), the polytrophic head coefficients are compared, and Fig. 8 compares the characteristics of

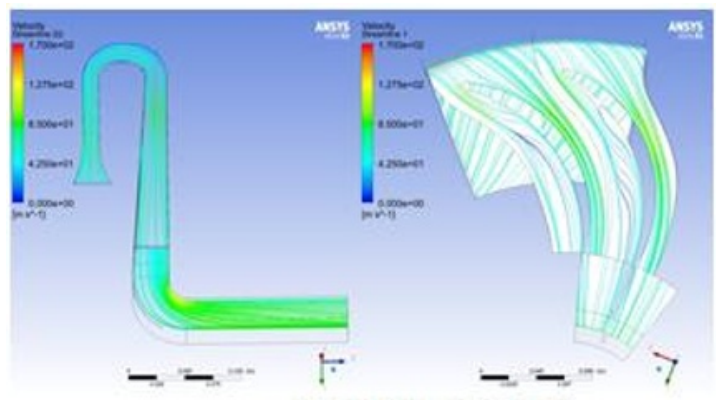

a) initial variant

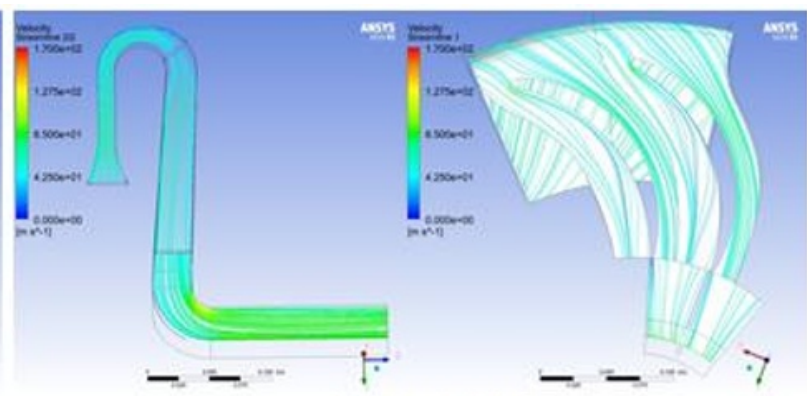

b) variant 3

Fig. 6. ANSYS CFX 19. Streamlines in the meridional (above) and radial (below) planes, $\Phi=0.0457$.
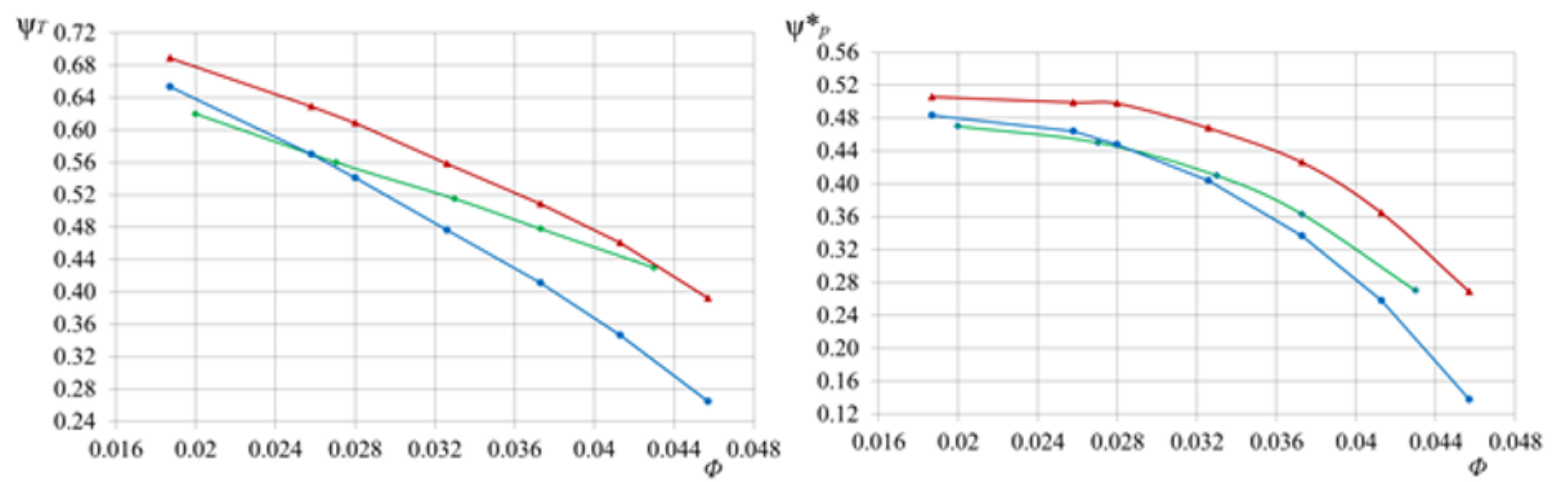

Fig. 7. Characteristic of the head coefficient (left) and the polytrophic head coefficient characteristic (right). Impeller 0028-0580373 (experiment) - green, impeller 0028-058-0373 (calculation) - red, impeller 0028-058-0373 with reduced $b_{2}$ (calculation) blue. 


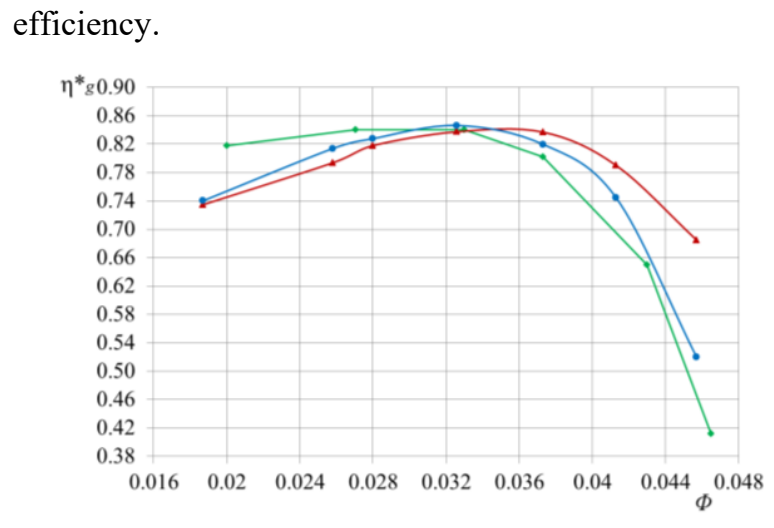

Fig. 8. Efficiency characteristic of the stage 0028-058-0373 (experiment) - green, impeller 0028-058-0373 (calculation) red, impeller 0028-058-0373 with reduced (calculation) blue.

The calculation of the stage with the initial variant of the stator elements and with the optimized variant 3 qualitatively confirmed the optimization result presented in the Fig. 5. Optimization of return channel slightly increased the efficiency and polytropic head coefficient at flow rates higher than the calculated one.

\section{Conclusion}

In this paper we have investigated the stator elements of the model stage 0028-058-0373 (design flow coefficient 0.028 , design loading factor 0.58 , hub to impeller ratio 0.373). Comparison of the initial and two-arc vane profiles showed a less favorable flow around the suction surface of the vane near the leading edge of the initial variant of the vane profile. The loss coefficient in the design mode of the two-arc profile is $0.2 \%$ higher. Optimization of return channel by five parameters allowed to reduce the values of the loss coefficient by $4.4 \%$ in the calculated mode and by up to $33 \%$ in the modes higher than the calculated one. The main influence on the efficiency was exerted by an increase in the vane height at the vane cascade inlet $b_{5}$ with a simultaneous decrease of the inlet vane angle $\alpha_{v 5}$. The best optimized variants have values $b_{5} / b_{4}=1.76 \ldots$ 2.08. The values of vane thickness $\delta / D_{2}=0.035 \ldots$ 0.04 , which is also higher than that of the initial variant. As a result of optimizing the crossover shape, the stator elements loss coefficient decreased by a small amount of about $1 \%$ in the design mode. The optimized variant has a larger sleeve radius $R_{h} / b_{4}=1.77$ compared with the initial $R_{h} / b_{4}=1.09$, which leads to an undesirable increase in the overall dimensions of the compressor. The principle of choosing the vane height $b_{6}$ based on the conditions of the necessary acceleration at the entrance to the next stage [1], at the same time ensures good efficiency of this section of return channel. The calculation of the characteristics of the stage with the initial and optimized return channel confirmed the somewhat greater efficiency of the optimized variant at flow rates greater than the designed one. The positive effect of optimization is small, which indicates a good level of design techniques presented in [1]. The authors believe that with the planned studies of stages with medium and large flow coefficients, optimization will give a more noticeable increase in efficiency.

\section{Acknowledgements}

The calculations were carried out using the supercomputer center Polytechnic, SPbPU. The research was performed by a Grant of the President of the Russian Federation for young PhD MK-1893.2020.8.

\section{References}

[1] K. Seleznev, Y. Galerkin, Centrifugal compressors (L.: mechanical engineering, 271, 1982)

[2] Yu. Galerkin, Turbocompressors (LTD information and publishing center KHT, Moscow, 596, 2010)

[3] R.H. Aungier, Centrifugal compressors: a strategy for aerodynamic design and analysis, The American Society of Mechanical Engeneers (2000)

[4] N.A. Cumpsty, Compressor Aerodynamics (Longman Scientific and Technical, England, 1989)

[5] C. Rube, T. Rossbach, M. Wedeking, D.R. Grates, P. Jeschke, Experimental and numerical investigation of the flow inside the return channel of a centrifugal process compressor, Turbine Technical Conference and Exposition, Montreal, Canada (2015)

[6] F.D. Bellis, E. Guidotti, D.T. Rubino, Centrifugal compressors return channel optimization by means of advanced 3D CFD, Turbine Technical Conference and Exposition, Montreal, Canada (2015)

[7] C. Aalburg, A. Simpson, J. Carretero, T. Nguyen, V. Michelassi, Extension of the stator vane upstream across the 180deg bend for a multistage radial compressor stage, Proceedings of ASME Turbo Expo 2009: Power for Land, Sea and Air, GT2009-59522 (8-12 June 2009)

[8] M. Yagi, T. Nishioka, H. Kobayashi, H. Nishida, S. Yamamoto, Effects of return channel with splitter vanes on performance of multistage centrifugal compressor, Turbine Technical Conference and Exposition, Montreal, Canada (2015)

[9] A. Veress, R. Van den Braembussche, Inverse design and optimization of a return channel for a multistage centrifugal compressor, Journal of Fluids Engineering 126, 799806 (2004)

[10] Y. Nishida, H. Kobayashi, H. Nishida, K. Sugimura, Performance improvement of a return channel in a multistage centrifugal compressor using multiobjective optimization, Journal of Turbomachinery 135, 031026, 1-8 (2013) 
[11] A. Hildebrant, Aerodynamic optimization of a centrifugal compressor return channel and U-turn with Genetic Algorithms, ASME Turbo Expo 2011, ASME Paper GT201145067 (2011)

[12] A. Hildebrandt, Numerical analysis of overall performance and flow phenomena of an automatically optimized treedimensional return channel system for multistage centrifugal compression systems, ASME Turbo Expo 2012, ASME Paper GT2012-68559 (2012)

[13] Yu.B. Galerkin, A.F. Rekstin, A.A. Drozdov, K.V. Soldatova, O.A. Solovyeva, E.Yu Popova, Through a system of optimal gas-dynamic design of industrial centrifugal compressors. Scientific basis, application practice, XVIII International Scientific and Technical Conference, Kazan (2019)

[14] V. Riess, Centrifugal compressors (1981)

[15] Yu.B. Galerkin, L.N. Marenina, Compressors and pneumatics 1, 30-36 (2014)

[16] L. Marenina, Y. Galerkin, K. Soldatova, Computational fluid dynamics application for analysis of centrifugal compressor stage stator part, International Journal of Mechanical Engineering and Robotics Research 7, 6, 656661. DOI: 10.18178/ijmerr.7.6.656-661 (2018)

[17] Yu.B. Galerkin, A.F. Rekstin, L.N. Marenina, K.V. Soldatova, Creation of parametrizes model of return channel flow path for CFD-researches, Oil and Gas Engineering-2020, Materials of the 10th International Scientific and Technical Conference, Omsk, 127-128 (2020)

[18] Y. Galerkin, K. Soldatova, Gas-dynamic process in industrial centrifugal compressors modeling, Scientific basis, stapes of development, current state, Publishing house of Polytechnic University, 327 (2011)

[19] Y. Galerkin, A. Rekstin, K. Soldatova, Gas dynamic design of the pipe line compressor with 90\% efficiency [Electronic resource], Model test approval, International Conference on Compressors and their Systems, London, City University, UK. Available at: http://iopscience.iop.org/article/10.1088/1757899X/90/1/012044/meta. DOI: $10.1088 / 1757$ 899X/90/1/012044 (2015)

[20] Y. Galerkin, A. Rekstin, K. Soldatova, A. Drozdov, Gas dynamic designs of centrifugal compressors for gas industry, Specific features, ASME Gas Turbine India Conference, GTINDIA2015-1215. Available at: www.asmeconferences.org/GTIndia2015/PaperA ccepted.cfm?noToolbar=yes. DOI: 10.1115/GTINDIA2015-1215 (2015)

[21] Y. Galerkin, K. Soldatova, A. Drozdov, The application of mathematical models for industrial centrifugal compressor optimal design, ICCMS 2018: 10th International Con-ference on Computer Modeling and Simulation, Australia, Sydney, 008, 187-191 (2018)
[22] A.F. Rekstin, V.B. Semenovskiy, Y.B. Galerkin, A.A. Sokolov, The analysis of design and measured gas-dynamic characteristics of the centrifugal compressor within turboexpander aggregate, Oil and Gas Engineering (OGE-2018), AIP Conf. Proc. 2007, Omsk, 030028-1-03002811. DOI: $10.1063 / 1.5051889$ (2018)

[23] A. Rekstin, V. Semenovskiy, K. Soldatova, Y. Galerkin, K. Sokolov, The simulation of gasdynamic characteristics of centrifugal compressors in turbo-expander units, International Scientific and Technical Conference Smart Energy Systems, SES 2019, Kazan, E3S Web of Conferences 124, 01005. DOI: 10.1051/e3sconf/201912401008 (2019)

[24] P. Le Sausse, P. Fabrie, D. Arnou, F. Clunet, CFD comparison with centrifugal compressor measurements on a wide operating range, EPJ Web of Conferences 45, 01059. DOI: 10.1051/epjconf /20134501059 (2013)

[25] A. Borovkov, I. Voinov, Y. Galerkin, A. Nikiforov, M. Nikitin, O. Solovyeva, K. Kabalyk, Issues of gas dynamic characteristics modeling: a study on a centrifugal compressor model stage, International Scientific Conference on Energy, Environmental and Construction Engineering (EECE-2019), E3S Web Conf. 140. DOI: 10.1051/e3sconf/201914006003 (2019)

[26] N. Kortikov, A. Borovkov, I. Voynov, A. Kirillov, A. Drozdov, Modeling the gas-dynamic characteristics of the low-flow and mid-flow model stages for an industrial centrifugal compressor, International Scientific Conference on Energy, Environmental and Construction Engineering (EECE-2018), MATEC Web Conf. 245. DOI: 10.1051/matecconf/201824504019 (2018) 